\title{
Simulating phase transition dynamics on nontrivial domains
}

\author{
Łukasz Bolikowski and Maria Gokieli \\ Interdisciplinary Centre for Mathematical and Computational Modelling \\ University of Warsaw \\ Prosta 69, 00-838 Warsaw, Poland \\ L.Bolikowski@icm.edu.pl, M.Gokieli@icm.edu.pl
}

\begin{abstract}
Our goal is to investigate the influence of the geometry and topology of the domain $\Omega$ on the solutions of the phase transition and other diffusion-driven phenomena in $\Omega$, modeled e.g. by the Allen-Cahn, Cahn-Hilliard, reaction-diffusion equations. We present FEM numerical schemes for the Allen-Cahn and Cahn-Hilliard equation based on the Eyre's algorithm and present some numerical results on split and dumbbell domains.
\end{abstract}

Keywords: diffusion, Cahn-Hilliard, Allen-Cahn, stability, finite element

\section{Introduction and motivation}

The Allen-Cahn equation:

$$
u_{t}-\varepsilon \Delta u=u-u^{3} \quad \text { on }(0, \infty) \times \Omega
$$

where $u=u(t, x), u(0, \cdot)=u_{0}$ given in some domain $\Omega$ and with the homogenous Neumann boundary condition

$$
\frac{\partial}{\partial n} u=0 \quad \text { on }(0, \infty) \times \partial \Omega,
$$

is of reaction-diffusion type. It is also a model of an order-disorder phase transition in alloys, $u$ being then not a concentration, but a non-conserved order parameter [1]. In any interpretation the equation is a dissipative system, driven by the minimization of the free energy

$$
\mathcal{F}=\int_{\Omega} \frac{\varepsilon}{2}|\nabla u(x)|^{2}+\frac{1}{4} u^{4}-\frac{1}{2} u^{2} d x
$$

in the Hilbert space $L^{2}(\Omega)$. It takes the so-called gradient form

$$
\frac{d u}{d t}=\frac{\delta \mathcal{F}}{\delta u}(u)
$$


the Gateaux derivative here being taken in $L^{2}(\Omega)$.

A second classical model of phase transition phenomena, also closely related to diffusion, is the Cahn-Hilliard equation [2, [3]. Roughly, it can be written as:

$$
u_{t}-\Delta\left(-\varepsilon \Delta u-u+u^{3}\right)=0 \quad \text { on }(0, \infty) \times \Omega
$$

$u(0, \cdot)=u_{0}$ given in $\Omega$ and with the boundary condition

$$
\frac{\partial}{\partial n} u=\frac{\partial}{\partial n} \Delta u=0 \quad \text { on }(0, \infty) \times \partial \Omega
$$

Here, $u$ is the concentration of one of the two components of an alloy or a mixture.

As shown by Fife 4, this equation is also a gradient system for the same free energy $\mathcal{F}$, but this times in the space $\tilde{H}^{-1}$, which the zero-average subspace of the dual of $H^{1}(\Omega)$.

The dynamics of both these equations are far from being trivial. One of their main characteristics is the coexistence very different time evolution scales: very fast and very slow. This is closely related to the attractor's structure of these systems, and in particular to their steady states. Let us give an example, for the simpler Allen-Cahn equation, considered in one space dimension only. Its steady states are then given by

$$
-\varepsilon u_{x x}+u^{3}-u=0 \quad \text { on }(a, b), \quad u^{\prime}(a)=u^{\prime}(b)=0 .
$$

Apart from constant solutions $0,-1$ and 1, there are many states which are close to 1 or -1 by the ends on the interval, and have one or more 'transition layers' or 'interfaces' between these almost constant states. If we understand by stability of these states their being or not 'attractive' for evolving solutions in their neighborhood, one gets immediately that 1 and -1 are stable and 0 is unstable (just considering the linearization of the left-hand-side operator around these states and its eigenvalues). It is known also that more layers in the steady state means more instability. The numerical experiments performed by Fusco and Hale in 1989 [5] seemed to indicate additionally stability of the one-layer solution. They discovered, however, by a theoretical study, that its apparent immobility was only an extremely slow evolution toward a constant state - the final transition occurring, on the contrary, instantaneously. The solution got the name of 'dormant instability'.

Things get more complicated when we pass to higher space dimensions. The influence of the domain's shape on the dynamics, and particularly on the character of the steady solutions, is extensively studied since the fundamental results of Casten and Holland [6] and of Matano [7. They found, independently, that in a class of regions including convex domains and annuli, the only stable solution of the reaction-diffusion type Neumann problems were constants. Other shapes have been considered from then on. The most studied one was the dumbbell, see Fig. 1 $(8$, , 9, 10, 11, [12, 13 - 14, 15]). Other shapes have been considered in [16]-17, 13]- 14], [15]. In [18] we studied this question domains, with 


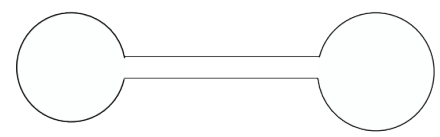

dumbbell domain

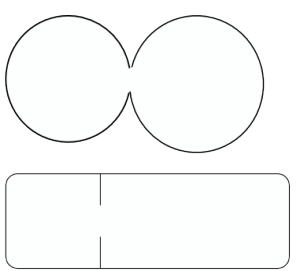

domains with a crack

Fig. 1. Some shapes for which the question of reaction-diffusion / Allen-Cahn dynamics have been studied theoretically.

a non-lipschitz boundary (see Fig. 1), obtaining existence of non-constant stable equilibria.

The same question appears for the Cahn-Hilliard equation, it has been studied e.g. in [19, 20], 21] [22, 223, although the domain shape influence is very little known for this case.

Let us also note that both equation coupled were also proposed as a model of two simultaneously occurring phase transitions in alloys [24].

Numerical simulations for phase transitions are still few, and inexistant, as far as we know, for complex domain shapes.

In [25] and [26], we proposed a numerical scheme and a solver for a system of both equations with a more sophisticated nonlinearity. Special cases included both equations taken separately. This fully implicit, nonlinear scheme was based on a splitting method of Lions and Mercier [27. Previous numerical studies of phase transitions (see e.g. [28], 29] and references therein for the method, 30], 31] for its application) were based on the same idea.

A much simpler and faster method, even if based on a similar splitting, has been proposed by Eyre [32] and exploited by Vollmayr-Lee and Rutenberg in 33. They show that with an appropriate choice of parameters, this scheme is semi-implicit (linearly implicit), and still stays a good approximation of the original equations. This method has been used to get a finite difference scheme on a square in [34, 35]. Actually, for simple rectangular domains this semi implicit scheme can be solved via the discrete cosine transform. This would be fast and effective, but inappriopriate for the object of our interest, i.e. complex domain shapes. This is why we turn to the free element method.

We thus apply here the idea of Eyre, Vollmayr-Lee and Rutenberg and to get a finite element scheme solving both equations (Section 2) and show some numerical results in split domains (Section 3). We hope that the conference will be an impulse to continue and broaden these first experiments. In a forthcoming [36] we will present a detailed proof of convergence and a solver for this method in a more general setting. 


\section{Discrete scheme}

We assume that $\Omega$ is polygonal. In $\Omega$ we introduce a family of quasi-uniform triangulations $T_{h}(\Omega)$ consisting of elements that are tetrahedrons in $3 \mathrm{D}$ case or triangles in $2 \mathrm{D}$ case, cf. 37] or Definition 4.4.13, p.106 in [38. We call the parameter of triangulation, and denote by $h$, the maximum over the diameters of all the triangles.

We work with the simple generic case of linear finite elements over $T_{h}(\Omega)$, i.e. we introduce the space

$$
V^{h}=\left\{v \in C(\bar{\Omega}): v_{\mid T} \in P_{1}(T) \forall T \in T_{h}(\Omega)\right\}
$$

where $P_{1}(T)$ is the set of linear polynomials over the element $T \in T_{h}(\Omega)$. The set of all nodal points, i.e. the vertices of elements of $T_{h}(\Omega)$, is denoted by $\Omega_{h}$.

\subsection{The scheme for Allen-Cahn}

We present the scheme for (1). Actually, the functions $u^{3}$ and $u$ could be replaced by any increasing, locally lipschitz functions, but let them be fixed for clarity. We use the scheme proposed in [33] with $a_{2}=0, a_{3}=1$ and $a_{1}=a$.

Let $\tilde{u}$ be the value from the previous time step. The unknown value $u$ is obtained from:

$$
\frac{u-\tilde{u}}{\tau}-\varepsilon \Delta u=(1-a) u+a \tilde{u}-\tilde{u}^{3}
$$

Translated into the finite element method, (3) is: knowing $\tilde{v}=v^{n-1} \in V^{h}$, find $v=v^{n} \in V^{h}$ such that for all test functions $\phi \in V^{h}$

$$
(1+(a-1) \tau) \int_{\Omega_{h}} v \phi+\tau \varepsilon \int_{\Omega_{h}} \nabla v \nabla \phi=(1+a \tau) \int_{\Omega_{h}} \tilde{v} \phi-\tau \int_{\Omega_{h}} \tilde{v}^{3} \phi
$$

This leads to a linear system of equations with a positive definite matrix, having a unique solution for all $n$. As shown in [33], for $a \geq 4$ this scheme is gradient stable, i.e. it has the property of conserving the gradient structure of the problem:

$$
\mathcal{F}\left(v^{n}\right) \leq \mathcal{F}\left(v^{n-1}\right)
$$

Another property which is important from the modeling point of view is the maximum principle: the solution stays in the interval $[-1,1]$.

The linear system if equations that we obtain from (4) can be solved by a direct or an iterative method. We actually use the direct Cholesky decomposition. This part can be parallelized. 


\subsection{The scheme for Cahn-Hilliard}

We re-write (2) as:

$$
\left\{\begin{array}{l}
u_{t}=\Delta w \\
w=-\varepsilon^{2} \Delta u+u^{3}-u
\end{array}\right.
$$

with the same boundary and initial conditions. The splitting allows to work with test functions taken only from $H^{1}(\Omega)$, and, in the discrete case, from $V^{h}$ - this means that we can use only standard $P_{1}$ finite elements.

The weak formulation of the problem is then:

$$
\left\{\begin{array}{l}
\int_{\Omega} u_{t} \varphi+\int_{\Omega} \nabla w \nabla \varphi=0 \\
\int_{\Omega} w \psi-\varepsilon^{2} \int_{\Omega} \nabla u \nabla \psi-\int_{\Omega}\left(u^{3}-u\right) \psi=0
\end{array}\right.
$$

which should be satisfied for all $\phi, \psi \in L^{\infty}\left(0, T ; H^{1}(\Omega)\right)$. See e.g. 25] for the details of the weak formulation.

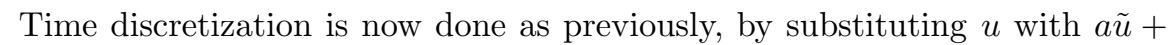
$(1-a) u$, where $\tilde{u}$ is the value from the previous time step. This gives the following scheme: knowing $\tilde{u}=u^{n-1} \in V^{h}$, find $(u, w)=\left(u^{n}, w^{n}\right) \in V^{h} \times V^{h}$ such that for all pairs of test functions $(\phi, \psi) \in V^{h} \times V^{h}$

$$
\left\{\begin{array}{l}
\int_{\Omega_{h}} \frac{u-\tilde{u}}{\tau} \varphi+\int_{\Omega_{h}} \nabla w \nabla \varphi=0 \\
\int_{\Omega_{h}} w \psi-\varepsilon^{2} \int_{\Omega_{h}} \nabla u \nabla \psi-\int_{\Omega_{h}}\left(\tilde{u}^{3}-a \tilde{u}-(1-a) u\right) \psi=0
\end{array}\right.
$$

or

$$
\left\{\begin{array}{l}
\int_{\Omega_{h}} u \varphi+\tau \int_{\Omega_{h}} \nabla w \nabla \varphi=\int_{\Omega_{h}} \tilde{u} \phi \\
\int_{\Omega_{h}} w \psi+(1-a) \int_{\Omega_{h}} u \phi-\varepsilon^{2} \int_{\Omega_{h}} \nabla u \nabla \psi=\int_{\Omega_{h}}\left(\tilde{u}^{3}-a \tilde{u}\right) \psi=0 .
\end{array}\right.
$$

This is again a semi-implicit scheme, leading to a linear system of equations, of unknown $(u, w) \in \mathbb{R}^{2 N}$, with a regular matrix:

$$
A=\left[\begin{array}{rc}
M & \tau G \\
(1-a) M-\varepsilon G & M
\end{array}\right]
$$

where $M$ is the mass matrix and $G$ is the stiffness matrix; $\operatorname{det} A=\operatorname{det}\left(M^{2}+\right.$ $\left.\tau(a-1) M G+\tau \varepsilon G^{2}\right)>0$, see the forthcoming [36] for details.

\section{$3 \quad$ Numerical experiments}

\subsection{Results for Allen-Cahn}

The computations for the Allen-Cahn equation has been performed with use of the FreeFEM software on a domain which approximates non-Lipschitz domains: 
we took two nearly circular subdomains and joined them by one node to which we add two more very small elements, as shown on Fig. 2. On the right-handside there, we show the smallest of all holes considered in our experiments. More experiments with finer meshes would still be needed. However, the results of simulations that we present below are in perfect accordance with theory established in 18 ; it is thus clear that no numerical artefacts are observed here.
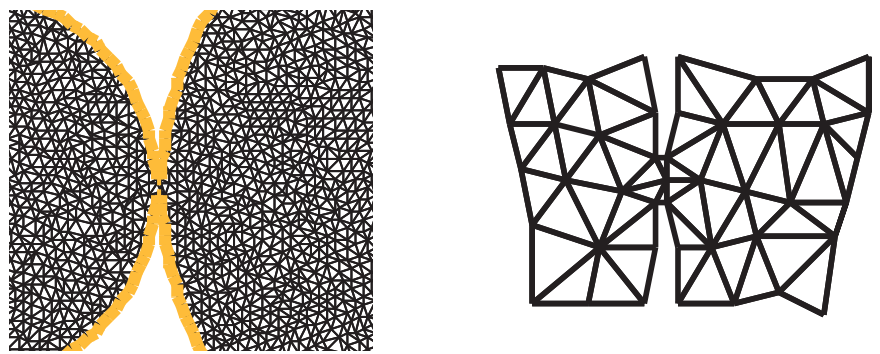

Fig. 2. Two close-up views on the FEM grid near the junction of the two subdomains.

Two experiments are shown in Figure 3 . They are performed with initial data taken as random perturbations of the constant 0 , the constant unstable solution. The system evolves towards two different states: a constant solution (equal to -1 ) in case (a) and a nonconstant one, close to \pm 1 in each subregion in case (b). None of this data seem to evolve. Whereas it is clear that the constant final state in (a) is stable, we continue the computations for (b) in order to check if any evolution towards a constant state can be seen. The non-constant state seems stable. This is actually true, as shown tn the theretical paper [18. However, for a numerical confirmation and an insight into the dynamics of the studied process, we plot a measure of the rate of change of the function $u$ in time, defined as:

$$
m\left(t_{n}\right)=\int_{\Omega} \frac{\left|u^{n}-u^{n-1}\right|}{\tau}
$$

Here $n$ is the time step, $u^{n}$ the numerical solution at time step $n$, i.e. at $t_{n}=n \tau$. The measure is more sensitive to changes of $u$ than the rate of change of mass (where mass is defined as $\int_{\Omega} u$ ), in particular: $m\left(t_{n}\right)=0$ implies $u^{n} \equiv u^{n-1}$ a.e. Figure 6 presents the measure for each of the experiments. Note that logarithmic scale is used for the vertical axes.

One can see that the evolution speed is (i) nearly constant but increasing on big intervals of time, and (ii) changing rapidly on some very short intervals. This phenomenon is known to correspond to the evolution on the attractor, (i) following the invariant manifolds and (ii) near the unstable equilibria (see for instance [5] for the Allen-Cahn case). Our graphs show also a much slower and flatter part by the end of all the experiments, which makes one think there is a particular feature of the final state - and this is actually its stability. 


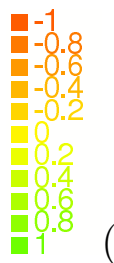

(a)
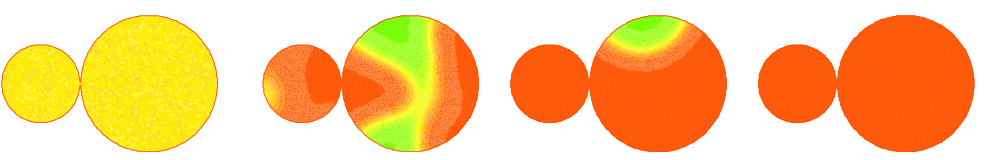

(b)
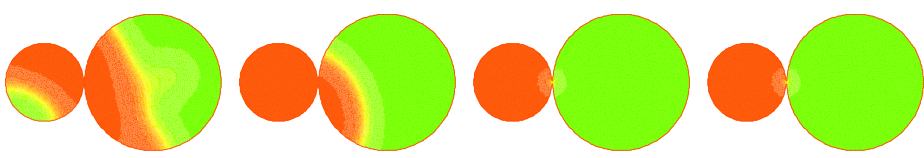

Fig. 3. Two experiments done with initial data $u_{0} \approx 0$, with random perturbations. The evolution is visualized at $t=0, t=0, t=10, t=40, t=100$ and $t=1000$ in (a) and $t=0, t=10, t=100, t=200, t=1000$ in (b). The legend stands for both experiments. The numerical parameters are time-step $\tau=0.001$, the diameter of the triangulation $h_{\max }=0.00175$. The physical parameter $\varepsilon=0.09$.

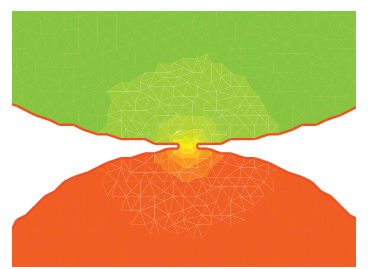

Fig. 4. The interface in the final stage from Fig. 3 (b), a close-up.

(a)

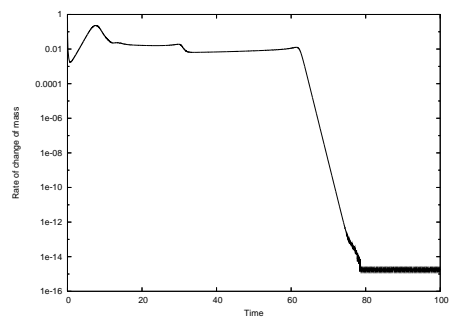

Fig. 5. The rate of mass change 8 for experiments of Figure 3

\subsection{Results for Cahn-Hilliard}

For the Cahn-Hilliard equation 2, experiments were performed on a dumbbell domain. Here, the process is much slower and we focused up to now on the 
experiments visualizing the spinodal decomposition and the nucleation processes. The first one is occurring very rapidly, which again corresponds to the vicinity of the steady state in the attractor. Then, we observe a very slow process of nucleation. It occurs at the same speed in the narrow channel and in the large subregions. The theoretical and numerical stability analysis is still to be done.

The experiment in Fig. 3.2 is performed with the numerical scheme 7 again using Free FEM.

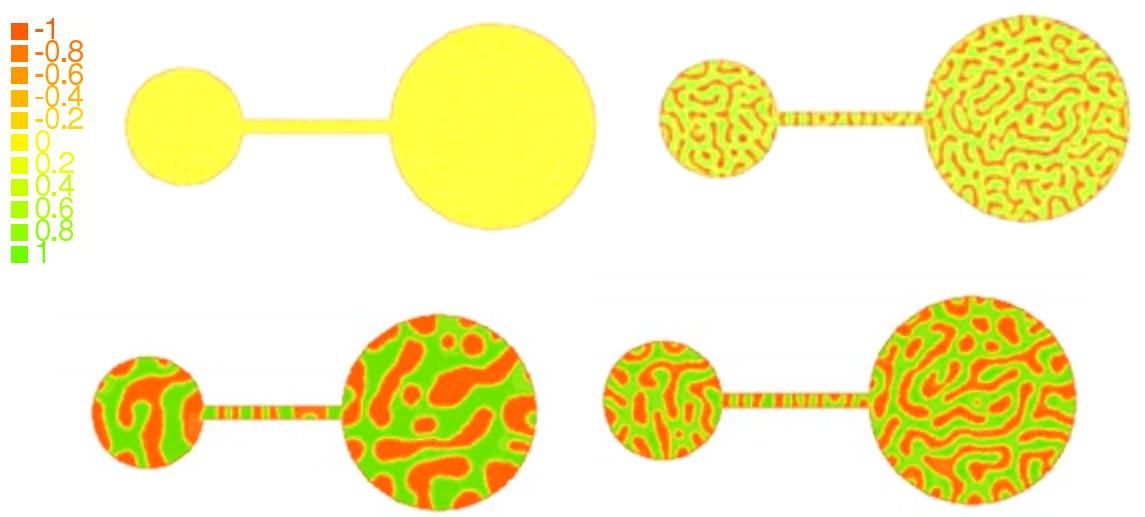

Fig. 6. The nucleation process for the Cahn-Hilliard equation. The geometry of the domain is similar to the previous one, but we add a channel between the two subregions. The evolution is visualized at $t=0, t=10, t=700, t=200, t=5000$. The legend stands for both experiments. The parameters are as before time-step $\tau=0.001$, the diameter of the triangulation $h_{\max }=0.00175, \varepsilon=0.09$.

\section{References}

1. Allen, S.M., Cahn, J.W.: A microscopic theory for antiphase boundary motion and its application to antiphase domain coarsening. Acta Metallurgica 27(6) (1979) 1085-1095

2. Cahn, J.W., Hilliard, J.E.: Free energy of a nonuniform system. i. interfacial free energy. J.Chem.Phys. 28(2) (1958) 258-267

3. Cahn, J.W., Hilliard, J.E.: Spinodal decomposition - a reprise. Acta Metall. 19 (1971) 151-161

4. Fife, P.C.: Models for phase separation and their mathematics. (2000)

5. Fusco, G., Hale, J.K.: Slow-motion manifolds, dormant instability, and singular perturbations. J. Dynam. Differential Equations 1(1) (1989) 75-94

6. Casten, R.G., Holland, C.J.: Instability results for reaction-diffusion equations with Neumann boundary conditions. J. Differ. Equations 27 (1978) 266-273 
7. Matano, H.: Asymptotic behavior and stability of solutions of semilinear diffusion equations. Publ. Res. Inst. Math. Sci. 15 (1979) 401-454

8. Hale, J.K., Vegas, J.: A nonlinear parabolic equation with varying domain. Arch. Ration. Mech. Anal. 86 (1984) 99-123

9. Vegas, J.M.: Bifurcations caused by perturbing the domain in an elliptic equation. J. Differ. Equations 48 (1983) 189-226

10. Jimbo, S.: The singularly perturbed domain and the characterization for the eigenfunctions with Neumann boundary condition. J. Differ. Equations 77(2) (1989) $322-350$

11. Jimbo, S.: Singular perturbation of domains and semilinear elliptic equations. III. Hokkaido Math. J. 33(1) (2004) 11-45

12. Jimbo, S., Morita, Y.: Remarks on the behavior of certain eigenvalues on a singularly perturbed domain with several thin channels. Commun. Partial Differ. Equations 17(3/4) (1992) 523-552

13. Arrieta, J.M., Carvalho, A.N., Lozada-Cruz, G.: Dynamics in Dumbbell domains. I: Continuity of the set of equilibria. J. Differ. Equations 231(2) (2006) 551-597

14. Arrieta, J.M., Carvalho, A.N., Lozada-Cruz, G.: Dynamics in Dumbbell domains. I: Continuity of the set of equilibria. J. Differ. Equations 231(2) (2006) 551-597

15. de Oliveira, L.A., Pereira, A.L., Pereira, M.C.: Continuity of attractors for a reaction-diffusion problem with respect to variations of the domain. (2005)

16. Dancer, E.: The effect of domain shape on the number of positive solutions of certain nonlinear equations. J. Differ. Equations 74(1) (1988) 120-156

17. Daners, D.: Dirichlet problems on varying domains. J. Differ. Equations 188(2) (2003) 591-624

18. Bolikowski, Ł., Gokieli, M., Varchon, N.: The Neumann problem in an irregular domain. Interfaces Free Bound. 12(4) (2010) 443-462

19. Alikakos, N.D., Fusco, G.: Slow dynamics for the Cahn-Hilliard equation in higher space dimensions: The motion of bubbles. Arch. Ration. Mech. Anal. 141(1) (1998) $1-61$

20. Alikakos, N., Fusco, G., Karali, G.: Motion of bubbles towards the boundary for the Cahn-Hilliard equation. Eur. J. Appl. Math. 15(1) (2004) 103-124

21. Alikakos, N., Bates, P.W., Fusco, G.: Slow motion for the Cahn-Hilliard equation in one space dimension. J. Differential Equations 90(1) (1991) 81-135

22. Bates, P.W., Fusco, G.: Equilibria with many nuclei for the Cahn-Hilliard equation. J. Differ. Equations 160(2) (2000) 283-356

23. Sander, E., Wanner, T.: Unexpectedly linear behavior for the Cahn-Hilliard equation. SIAM J. Appl. Math. 60(6) (2000) 2182-2202 (electronic)

24. Cahn, J.W., Novick-Cohen, A.: Evolution equations for phase separation and ordering in binary alloys. J. Stat. Phys. 76(3-4) (1992) 877-909

25. Gokieli, M., Marcinkowski, L.: Discrete approximation of the Cahn-Hilliard/AllenCahn system with logarithmic entropy. Japan J. Ind. Appl. Math. 20(3) (2003) 321-351

26. Gokieli, M., Marcinkowski, L.: A solver for the finite element approximation scheme for the Cahn-Hilliard/Allen-Cahn system with logarithmic entropy. Aiki, T. (ed.) et al., Current advances in nonlinear analysis and related topics. Tokyo: Gakkōtosho. GAKUTO International Series. Mathematical Sciences and Applications 32 (2010)

27. Lions, P.L., Mercier, B.: Splitting algorithms for the sum of two nonlinear operators. SIAM J. Numer. Anal. 16 (1979) 964-979

28. Elliott, C.M., French, D.A.: A nonconforming finite element method for the twodimensional Cahn-Hilliard equation. SIAM J. Numer. Anal. 26(4) (1989) 884-903 
29. Blowey, J.F., Copetti, M.I.M., Elliott, C.M.: Numerical analysis of a model for phase separation of a multi-component alloy. IMA J. Numer. Anal. 16(1) (1996) 233-280

30. Kay, D., Welford, R.: A multigrid finite element solver for the cahn-hilliard equation. Journal of Computational Physics 212(1) (2006) 288-304

31. Wells, G.N., Kuhl, E., Garikipati, K.: A discontinuous galerkin method for the cahn-hilliard equation. Journal of Computational Physics 218(2) (2006) 860-877

32. Eyre, D.J.: An unconditionally stable one-step scheme for gradient systems. Preprint (1997), University of Utah, Salt Lake City

33. Vollmayr-Lee, B.P., Rutenberg, A.D.: Fast and accurate coarsening simulation with an unconditionally stable time step. Physical Review E 68(6) (2003) 066703

34. De Mello, E., Teixeira da Silveira Filho, O.: Numerical study of the cahn-hilliard equation in one, two and three dimensions. Physica A: Statistical Mechanics and its Applications 347 (2005) 429-443

35. Cheng, M., Rutenberg, A.D.: Maximally fast coarsening algorithms. Physical Review E 72(5) (2005) 055701

36. Bolikowski, Ł., Gokieli, M., Marcinkowski, L.: The Eyre's algorithm for solving phase transition models - analysis and application. in preparation

37. Quarteroni, A., Valli, A.: Numerical Approximation of Partial Differential Equations. Springer-Verlag, Berlin (1994)

38. Brenner, S.C., Scott, L.R.: The mathematical theory of finite element methods. Volume 15 of Texts in Applied Mathematics. Springer-Verlag, New York (1994) 